Rodríguez Álvarez, P.; Ocampo Gómez, C.I. y Sarmiento Campos, J.A. (2018). Valoración de la orientación profesional en la enseñanza secundaria postobligatoria. Revista de Investigación Educativa, 36(1), 75-91.

DOI: http://dx.doi.org/10.6018/rie.36.1.285881

\title{
Valoración de la orientación profesional en la enseñanza secundaria postobligatoria
}

\section{Assessment of professional guidance in post-compulsory secondary education}

\author{
Pablo Rodríguez Álvarez, Camilo Isaac Ocampo Gómez y José Antonio Sarmiento Campos \\ Departamento de Didáctica, Organización Escolar y Métodos de Investigación \\ Facultad de Educación \\ Universidad de Vigo (España)
}

\begin{abstract}
Resumen
La orientación académica y profesional es cada vez más un elemento clave en la formación a lo largo de la vida de las personas. La forma de llevar a cabo esa orientación y su éxito dependerá en gran parte de los modelos organizativos que adopten las diferentes instituciones para hacer más efectiva la intervención. Este trabajo pretende conocer con claridad la opinión del alumnado de Galicia sobre la orientación académica y profesional recibida en la enseñanza secundaria postobligatoria y también la importancia atribuida a la misma. El método de investigación fue de carácter descriptivo y exploratorio mediante encuesta a una muestra representativa de 2408 estudiantes de primer curso universitario. Los resultados evidencian una distancia significativa entre la orientación recibida y su importancia, siendo las puntuaciones en esta última significativamente más elevadas en todas las variables. Puede concluirse que la orientación es un factor de gran importancia y que el alumnado está poco satisfecho con el trabajo realizado en los centros de enseñanza secundaria postobligatoria. Se necesita más actividad grupal e individual, así como intervención con recursos convencionales y tecnológicos. La infrautilización de estos últimos es destacable en nuestro estudio.

Palabras clave: orientación profesional; orientación vocacional; servicio de orientación; orientación pedagógica; orientación.
\end{abstract}

Correspondencia: pablorodriguezalvarez@uvigo.es; cocampo@uvigo.es; sarmiento@uvigo.es. Pavillón 1, Campus As Lagoas, 32004, Ourense. 


\begin{abstract}
Professional guidance is increasingly a key element in lifelong learning. The way in which such orientation and success will be achieved will largely depend on the organizational models adopted by the different institutions to make the intervention more effective. This paper aims to know clearly the opinion of the Galician students about the professional guidance received in post-compulsory secondary education and also the importance attributed to it. The research method was descriptive and exploratory by means of a survey of a representative sample of 2408 first year university students. The results show a significant distance between the guidance received and its importance, with the scores being significantly higher in all variables. It can be concluded that guidance is a factor of great importance and that students are not satisfied with the work done in post-compulsory secondary schools. It requires more individual and group activity, as well as intervention with conventional and technological resources. The underutilization of the latter is noteworthy in our study.

Keywords: professional guidance; vocational guidance; guidance service; pedagogical guidance; guidance.
\end{abstract}

\title{
Introducción
}

En la actualidad podemos afirmar con contundencia que la orientación académica y profesional es un elemento clave en la educación, la formación y el asesoramiento a lo largo de la vida de las personas. En este sentido, las diferentes instituciones con peso en materia educativa se sirven de determinados modelos organizativos para hacer más efectiva la intervención (Álvarez González, 2009). Así, se puede concretar una estructura organizativa a nivel Europeo, de Estado, de Comunidad Autónoma (CCAA), población, etc.

Nuestro estudio se sitúa en la misma línea de muchos autores (Álvarez González, 1995, 2004; Álvarez Rojo et al., 2002; Barreira, 2002; García-Murias, Sobrado \& Fernández-Rey, 2016; Mariño, 2012; Ocampo, 2003; Ocampo, Sarmiento \& Barreira, 2016; Planas, 2009; Rodríguez Moreno 2002; Sanz Oro, 1999; Sobrado \& Ocampo, 2000; Velaz de Medrano, Manzano, López Martín, \& Manzano Soto, 2013; etc.) que se han preocupado de analizar los modelos organizativos-institucionales en las diferentes Comunidades Autónomas, en España y en Europa, tanto en el ámbito universitario como no-universitario. La principal conclusión extraída del análisis es que en las últimas décadas, se han producido numerosos e importantes avances en la institucionalización e implantación de la orientación educativa en España. Para Europa (Rodríguez Moreno, 1998; Sobrado, 1999; Sobrado \& Barreira, 2012; Watts et al., 1993; entre otros) los resultados son también concluyentes: existen modelos organizativos de orientación diferentes debido en gran parte a su historia distinta en materia educativa y laboral, e igualmente no hay una capacitación idéntica de los orientadores ni de las estructuras administrativas. No obstante y desde el punto de vista internacional, el nuevo escenario orientador puede definirse a partir del marco establecido por la Asociación Internacional de Orientación Educativa y Profesional (AIOEP), preocupada por el desarrollo de las competencias necesarias para pro- 
porcionar servicios de orientación educativa y profesional de calidad. Su propuesta compuesta por unas competencias generales y otras específicas y validada por 700 profesionales de 38 países, se adoptó en la Asamblea General en Berna en Septiembre de 2003. Más adelante, en el congreso de Copenhague (2006) se presenta la credencial del Orientador Educativo y Profesional, que supone un modo de visibilizar esas competencias. En un escenario parecido se sitúa el Centro Europeo para el Desarrollo de la Formación Profesional (CEDEFOP) en el 2009 al examinar las tendencias en la formación de los orientadores profesionales y desarrollar un marco de competencias comunes para éstos en el marco de la Unión Europea.

La Orientación entendida como una necesidad de cualquier persona adquiere una especial significación a lo largo del proceso de escolarización, constituyéndose como un derecho del alumnado y un factor de calidad que las administraciones han de garantizar en todos sus niveles. En este sentido en España con la promulgación de la Ley 14/1970, de 4 de agosto, General de Educación y financiamiento de la Reforma Educativa (LGE), se establece el derecho de los alumnos a la orientación educativa y profesional y en su artículo 125.2 se considera a ésta como un servicio continuado a lo largo de toda la vida escolar. Se destaca la función tutorial como elemento integrante de la función docente y en el artículo 127 se deduce la intención de ofrecer ayuda al alumnado en las tres vertientes de la orientación: escolar, personal y profesional. Para el Bachillerato se prevé la organización de actividades de orientación en las que el alumnado vea facilitada su orientación vocacional y en el art. 35.2 se establece el consejo orientador o asesoramiento para el Curso de Orientación Universitaria (COU), que serán sugerencias ofrecidas al alumnado para la elección de la carrera.

La Ley Orgánica 8/1985, de 3 de julio, reguladora del derecho a la educación (LODE), reconoce también el derecho de los alumnos a la orientación educativa y profesional y que corresponde al claustro de profesores la coordinación de las funciones de orientación y tutoría de los alumnos (Art. 45.2.d). Para Ocampo (2010), la LODE pone las bases (modelo de proyectos y programas) para que, luego, con la promulgación de la Ley Orgánica 1/1990, de 3 de octubre, de Ordenación General del Sistema Educativo (LOGSE), su aspecto organizativo (modelo de proyectos y programas), que permanece actualmente, sea el elemento principal del nuevo modelo institucional de la orientación educativa y profesional existente en los centros públicos en España y que, con diferencias propias de las concreciones que cada Comunidad Autónoma incorpora por razón de sus competencias en materia educativa, puede esquematizarse en los siguientes términos: departamento de orientación y tutoría como servicios internos y, además, equipos especializados externos, llevando a cabo, en conjunto, tareas de acuerdo con los principios de planificación y evaluación dentro de los proyectos y programas de cada centro.

Una vez promulgada la LOGSE podemos afirmar que al orientador se le entiende ya como un profesional del sistema educativo. Así la propia Ley ya hace referencia en su Preámbulo y primeros artículos a la actividad orientadora. En su Preámbulo la tutoría y la orientación se incluyen como parte de la función docente, estableciéndose el derecho del alumnado a orientación en los campos psicopedagógico y profesional. 
En definitiva, de los documentos que la preceden, de la propia LOGSE y de su desarrollo normativo posterior se extrae lo siguiente en relación a la orientación académica y profesional y sus tres niveles (Ocampo, 2010):

- En la tutoría se ha de promover el conocimiento personal (autoestima, aptitudes, intereses, motivaciones), el análisis de las diferentes salidas profesionales (con sus ventajas e inconvenientes), la colaboración con el departamento de orientación para conocer las opciones y el mercado laboral, y la implicación de los padres en la labor orientadora.

- A nivel departamental, se requiere que el centro asuma su labor orientadora profesional, así como la relación continúa con el mundo del trabajo. En este mismo nivel, se ofrece la ayuda en los momentos de riesgo e información sobre salidas académicas y profesionales.

- El equipo sectorial ha de proporcionar ayuda técnica al departamento y a la acción tutorial para poder desarrollar aquellas actividades vinculadas a la orientación profesional.

Estos tres niveles deben converger en la elaboración de los documentos que contienen los planes y programas de orientación y tutoría de un centro, así como a su aplicación. Cabe destacar el Plan de Acción Tutorial (PAT) y el Plan de Orientación Académica y Profesional (POAP), en concreto, el POAP, que es el que más se relaciona con la Orientación Profesional. Algunas características de las que presenta son:

- Tiene en cuenta las características personales de los alumnos y su entorno.

- Es facilitador de la toma de decisiones del alumno, conduciéndole libremente a su decisión personal en planes y proyectos.

- Propicia la actividad y la autonomía del alumno.

La Ley Orgánica 10/2002, de 23 de diciembre, de Calidad de la Educación (LOCE), mantiene el esquema del sistema educativo establecido por la LOGSE pero se convirtió en una ley relámpago por su escasa duración y aplicación, pues en el 2006 es sustituída por un gobierno de distinto signo politico por una nueva ley educativa denominada Ley Orgánica 2/2006, de 3 de mayo, de Educación (LOE), cuyos aspectos más formales comienzan a aplicarse en el curso 2006/07 y queda totalmente implantada en el 2009. Se mantienen expresamente los tres niveles establecidos por la LOGSE, a los que apenas se hacía referencia en la LOCE.

La Ley Orgánica 8/2013, de 9 de diciembre, para la Mejora de la Calidad Educativa (LOMCE), es una modificación de la vigente LOE (Ley Orgánica de Educación), cuya redacción se justifica en los resultados de las evaluaciones realizadas por organismos internacionales (y citados en ella como el informe PISA 2012 o el Programa Internacional para la Evaluación de la Competencia de los Adultos o PIAAC, conocido también como el PISA para adultos), con el fin de converger con los objetivos europeos en educación de la Estrategia Europa 2020. En esta ley no se contempla consideración específica directa alguna en relación a la orientación, quedando establecido el sistema 
orientador tal y como estaba desarrollado anteriormente a partir de la LOGSE y la LOE. Los cambios que afecten al sistema de orientación educativa en un futuro serán los que se deriven de su desarrollo legislativo.

La situación actual de la orientación en las Comunidades Autónomas Españolas una vez asumidas las competencias en materia educativas, es la resultante de haber establecido nuevos marcos legales de referencia en relación con los aspectos organizativos y funcionales de la orientación, conllevando la necesidad de adaptación de los servicios de orientación y dando lugar a modelos organizativos diferentes. Sánchez García (2013) explica que en la educación primaria y secundaria (subsistema de orientación escolar, para diferenciarlo del subsistema universitario y del subsistema sociolaboral) es donde la práctica orientadora está más desarrollada, institucionalizada, especializada e investigada. Su referente es el modelo institucional establecido en la LOGSE (1990) que se centra en el aprendizaje, en menos medida se desarrolla una orientación familiar y profesional, y se estructura en tres partes: en el aula a través del Plan de Acción Tutorial (PAT), en el centro a través del Departamento de Orientación (DO) y del Plan de Orientación Académica y Profesional (POAP), y en el sector o zona mediante los Equipos externos (con diferentes denominaciones según la comunidad autónoma de referencia).

Galicia en el ámbito de la orientación sigue pautas análogas a las del resto del Estado Español, así en el año 1998 y adaptándose a la LOGSE se promulga la nueva normativa que es la que rige actualmente en la Comunidad para los centros de enseñanza de niveles no universitarios: Decreto 120/1998, de 23 de Abril y Orden de 24 de Julio de 1998 que lo desarrolla. Se establecen unos servicios orientadores internos (Tutorías y Departamentos de Orientación, tanto en centros de educación infantil y primaria, como en institutos de educación secundaria y centros públicos integrados) y unos servicios orientadores externos, configurados por los Equipos de Orientación Específicos (continuadores en cierto modo de los Equipos Psicopedagógicos de Apoyo (que desenvolvían sus funciones en los centros públicos de los niveles no universitarios).

Las funciones a realizar por dichos departamentos, son muy similares independientemente de la etapa de referencia, no obstante en los niveles de infantil y primaria adquieren una mayor relevancia aquellas funciones relacionadas con la orientación educativa, mientras que en educación secundaria además de la orientación educativa tiene una gran importancia la orientación profesional. Como norma general es un orientador por centro independientemente del número de etapas educativas y/o alumnado del centro (no existe ratio orientador-alumnado), ejerciendo este además las funciones de jefe de departamento.

Por todo lo expuesto, cabe decir que el modelo institucional vigente, visto desde los principios teóricos de la orientación presenta aciertos importantes y supone un avance considerable en el desarrollo de la orientación en los centros docentes. Así por ejemplo en Galicia a partir del año 1998 se solventa eficazmente la presencia de los orientadores en los centros educativos, aunque no se regula la incorporación de estos a las aulas ni tampoco la atención directa que estos puedan prestar al alumnado en sus diversas necesidades. Entre ellas está lógicamente la necesidad de recibir orientación académico-profesional. En este sentido, el conocimiento empírico del hecho orientador 
nos lleva a pensar que la organización y el funcionamiento de los servicios de orientación pueden no estar del todo adecuados a la realidad educativa de los institutos de educación secundaria. Así por ejemplo, hoy en día en Galicia, nos encontramos con horarios lectivos que excluyen la posibilidad de tener contacto personal con los alumnos y alumnas de nuestros institutos, excesivo individualismo o modelos de trabajo personales en el día a día de los departamentos de orientación de los centros, excesiva asunción de funciones y gran diversidad de las mismas, inexistencia de un foro centralizado de participación y normativización de las tareas orientadoras, inexistente tutoría de expertos para profesionales de la orientación noveles y no tan noveles, etc.

¿Entonces?

Como orientadores e investigadores sentimos la obligación de hacer algo para conocer cuál puede ser la valoración de los destinatarios de la orientación en relación al modelo existente. Para ello planteamos un estudio que nos aporte información sobre la atención que recibe el alumnado por parte de los servicios de orientación en el área académico-profesional. Nos centramos en el alumnado de Galicia de enseñanza secundaria postobligatoria y no contamos con ningún estudio realizado específicamente con este alumnado que nos permita conocer con claridad lo que piensa este colectivo sobre la orientación recibida durante sus estudios de bachillerato y formación profesional en la Comunidad Autónoma de Galicia.

\section{Método}

\section{Objetivos}

Con el fin de valorar el modelo de intervención en orientación académica y profesional desarrollado en Galicia en la enseñanza secundaria postobligatoria, nos planteamos a partir de la opinión del alumnado los siguientes objetivos:

- Identificar el grado de materialización de determinadas actividades y/o la utilización de determinados recursos de orientación académica y profesional.

- Conocer la importancia que el alumnado le atribuye a las actividades y/o recursos utilizados.

- Analizar el contraste existente entre el grado de materialización de esas actividades y/o recursos utilizados con la importancia atribuida por el alumnado a los mismos.

Es un estudio sobre "algunos aspectos del proceso orientador" (Álvarez, 1995, p. 429) para aportar nueva información de cara a la intervención en los procesos de orientación académica y profesional, optimizar la orientación y mejorar su calidad y su eficacia.

\section{Población y Muestra}

El estudio se dirigió a una muestra representativa de alumnos y alumnas que ya habían experimentado el proceso "completo" de orientación académica y profesional propio de los estudios de secundaria postobligatoria. Es decir, buscamos alumnado 
con decisiones vocacionales ya superadas y que fuera capaz de opinar sobre los procesos de orientación recibidos. Así, durante el trabajo de campo se encuestó a 2408 estudiantes matriculados en primer curso universitario de 35 grados oficiales distintos de las tres universidades públicas de Galicia, lo que supone el $20.6 \%$ de la población investigada $(\mathrm{N}=11684)$. La muestra está formada por un $59.2 \%$ de mujeres (1425) frente al 40.8\% (983) de hombres, siendo la gran mayoría menores de 20 años $(84.37 \%)$.

\section{Instrumento}

Se trata de un estudio de carácter descriptivo y exploratorio. Mediante método de encuesta a través de un cuestionario anónimo elaborado ad hoc, recogemos las concepciones de los estudiantes de primer curso universitario de Galicia sobre la orientación académica y profesional recibida en la enseñanza postobligatoria. Cada ítem del cuestionario recoge un ambiente de trabajo y/o recurso utilizado admitiéndose una doble respuesta, una referida a la realización de la actividad y/o recurso planteado desde la propia experiencia del estudiante y otra a la importancia que este le atribuye al mismo. En dicho cuestionario se le pedía a los estudiantes que respondiesen a diferentes cuestiones dobles (elección doble) en relación a una lista de diversos ambientes propios de la orientación en la secundaria postobligatoria: Actividades, recursos, tecnología y temporalización empleados para la orientación. De este modo, su primera opción de respuesta tiene que ver con la orientación recibida (EXPERIENCIA personal o REALIDAD de la orientación) y su segunda opción tiene que ver con la importancia que le dan a ese hecho concreto (IMPORTANCIA de la orientación).

Superada una fase piloto o de pruebas y con el fin de garantizar la validez de contenido, el cuestionario se sometió a juicio de expertos mediante la aplicación de la Técnica Delphi a un plantel de profesionales constituido en su mayoría por orientadores y orientadoras educativos de centros públicos de enseñanza secundaria de Galicia. Tomando en consideración sus valoraciones en relación al diseño, la complejidad y coherencia de las cuestiones, se elaboró el cuestionario definitivo. La fiabilidad del cuestionario, estimada como consistencia interna a partir del alpha de Cronbach, fue de 0.931 .

Cada ítem del cuestionario admitía una doble respuesta (Realización o Importancia) mediante escalas tipo Likert de 0 a 4 puntos:

- Realización: 0 (No, nada, nunca), 1 (Sí, alguna que otra vez), 2 (Sí, varias veces), 3 (Sí, muchas veces), 4 (Sí, muchísimas veces o Siempre).

- Importancia: 0 (No es importante), 1 (Es poco importante), 2 (Es importante), 3 (Es muy importante), 4 (Es extremadamente importante).

Los items del cuestionario (Tabla 1) se agruparon en categorías más amplias según su contenido: actividades realizadas con el alumnado, actividades realizadas con las familias, recursos generales empleados, recursos telemáticos empleados, material aportado y horario de orientación a libre disposición del alumnado. 
Tabla 1

Apartados del cuestionario aplicado.

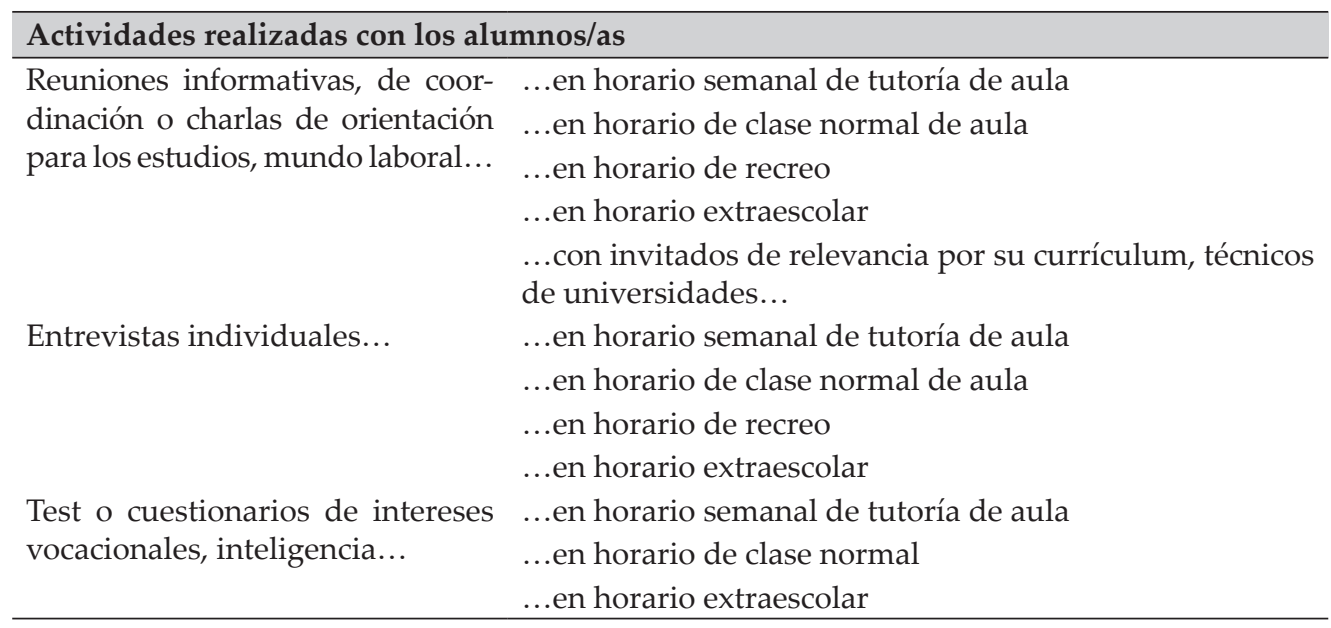

\section{Actividades realizadas con las familias}

Reuniones informativas, de coor- ...de orientación en general

dinación o charlas ...

...con invitados de relevancia por su currículum, técnicos de universidades...

Entrevistas individuales

\section{Recursos generales empleados}

PC con proyector (presentaciones en P. Point, httml...)

Pizarra digital

Encerado clásico

Internet: para enseñar portales web de universidades, becas, centros de estudios...

Tablón de anuncios a la vista de todos con información relevante: notas de corte, centros de estudios, universidades públicas y privadas...

Material de consulta: disponibilidad de manuales informativos sobre carreras, de ciclos formativos, Cds y Dvds, etc.

Cartelería, reportajes y fotografías...de empresas, centros de estudios especiales, universidades, asociaciones, ....con anuncios o publicidad variada

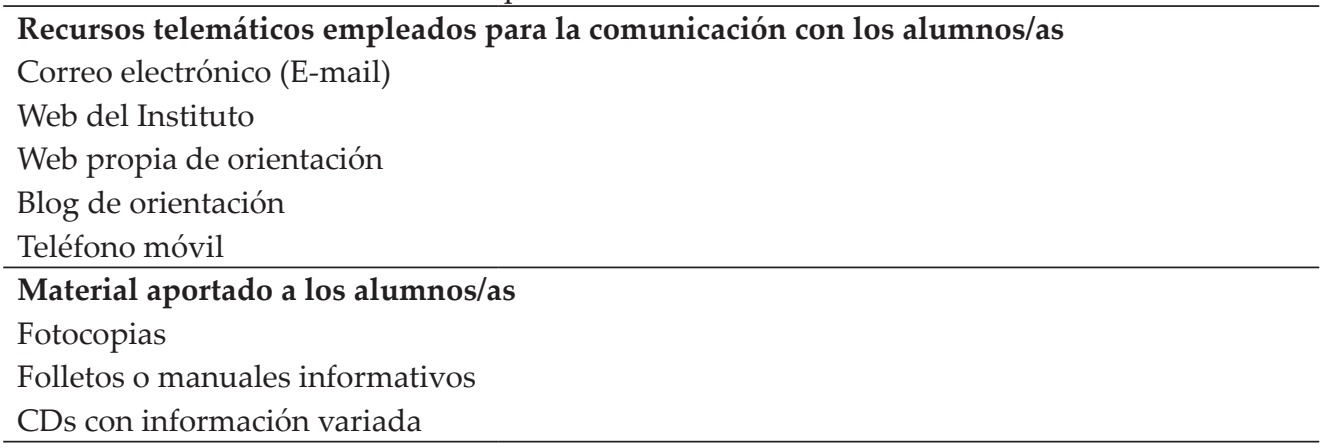




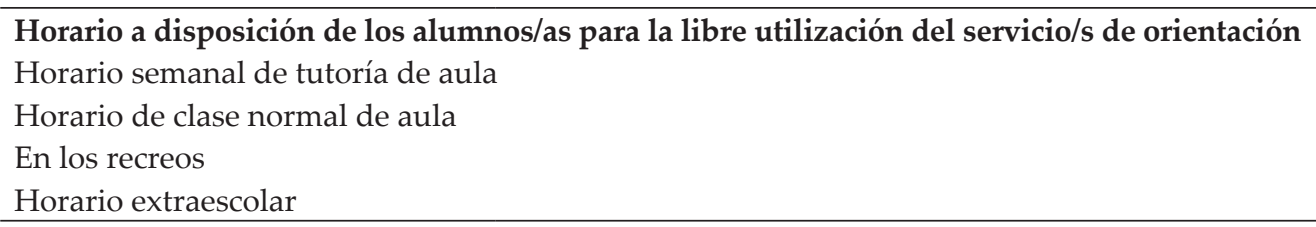

Fuente: Elaboración propia.

\section{Procedimiento de recogida de información y análisis de datos:}

Una vez delimitada la investigación y elaborado el instrumento, la recogida de información se llevó a cabo en el año 2011 en las aulas de 35 titulaciones universitarias distintas y pertenecientes a las 3 universidades públicas de Galicia. Una vez recogida la información y construida la base de datos se realizó el análisis de los mismos mediante el paquete estadístico SPSS (versión 17 a 20). Respecto a los dos primeros objetivos, para el análisis de las opiniones del alumnado, se efectuaron los cálculos de estadísticos descriptivos habituales en las ciencias sociales. Respecto del tercer objetivo se realizó un análisis de diferencias significativas mediante procedimientos no paramétricos observando los contrastes entre opiniones (Prueba de los rangos con signo de Wilcoxon).

\section{Resultados}

\section{Respecto del grado de materialización de determinadas actividades y/o la utilización de determinados recursos de orientación académica y profesional, así como de la importancia que el alumnado le atribuye a las mismas}

En relación a las reuniones llevadas a cabo de tipo informativo, de coordinación o charlas de orientación con los alumnos/as para orientarlos hacia diferentes estudios, mundo académico y laboral, etc.:

En horario de tutoría son importantes para un $82.65 \%$, pero un $34.35 \%$ manifiesta no haber tenido nunca una charla de este tipo en ese horario y un $38.56 \%$ dice haberlas tenido alguna que otra vez.

- En horario de clase normal (horario lectivo ordinario) son importantes para un $75.25 \%$, nunca las han recibido un $25.85 \%$ y un $43.78 \%$ alguna vez.

- En horario de recreo son poco o nada importantes para un $54.28 \%$, nunca las han recibido un $59.17 \%$ y un $24.37 \%$ alguna que otra vez.

- En horario extraescolar son importantes para un 52.52\%, nunca las han recibido un $68.18 \%$ y un $18.27 \%$ alguna que otra vez.

- Con invitados de relevancia son importantes para un $89.44 \%$, nunca las han recibido un $32.85 \%$ y un $33.76 \%$ alguna que otra vez.

En relación a las entrevistas individuales llevadas a cabo con los alumnos y las alumnas:

- En horario de tutoría son importantes para el $77.85 \%$, nunca las han tenido un $60.2 \%$ y un $23.28 \%$ alguna vez. 
- En horario de clase normal (horario lectivo ordinario) son importantes para un $64.45 \%$, nunca las han tenido un $62.78 \%$ y un $20.60 \%$ alguna vez.

- En horario de recreo son importantes para un $52.84 \%$, nunca las han tenido un $58.42 \%$ y un $22.70 \%$ alguna que otra vez.

- En horario extraescolar son importantes para un $55.37 \%$, nunca las han tenido un $74 \%$ y un $14.45 \%$ alguna que otra vez.

En relación al empleo con el alumnado de test o cuestionarios de intereses vocacionales, inteligencia, etc.:

- En horario de tutoría son importantes para el $75.44 \%$, nunca han tenido este servicio un $45.34 \%$ y un $30.99 \%$ alguna vez.

- En horario de clase normal (horario lectivo ordinario) son importantes para un $68.35 \%$, nunca han tenido el servicio un $42 \%$ y un $31.69 \%$ alguna vez.

- En horario extraescolar un 52.50\% no lo considera importante, nunca han tenido el servicio el $82.63 \%$ y un $10.23 \%$ alguna que otra vez.

En relación a las actuaciones con las familias:

Un $74.81 \%$ del alumnado considera importante tener charlas de orientación con las familias, pero un $41.45 \%$ manifiesta que nunca se realizaron y un $30.50 \%$ alguna que otra vez. Si estas son con personajes de relevancia invitados son importantes para el $70.77 \%$, aunque nunca se realizaron en el $60.90 \%$ de los casos, y alguna que otra vez en el $20.71 \%$ de los casos. En relación a las entrevistas individuales con las familias un $72.35 \%$ las considera importantes, pero nunca se realizaron en un $54.85 \%$ de los casos y alguna que otra vez en un $24.94 \%$ de los mismos.

En relación al empleo con el alumnado de determinados recursos, materiales y horarios. Recursos generales empleados:

Los resultados muestran infrautilización de determinados recursos tecnológicos como la pizarra digital. Así, un $64.20 \%$ de los encuestados manifiestan no haber observado la utilización de la pizarra digital en las distintas actividades de orientación de las que fueron objeto (Ver Tabla 2). Por otro lado y en contraposición (comparación tecnológica) al dato anterior destaca la gran utilización del "encerado clásico". Un $45.40 \%$ de los encuestados manifiestan ser este el recurso más extremadamente utilizado.

En cuanto al grado de importancia de los recursos expuestos, podemos afirmar a la vista de los datos que la mayoría de los encuestados les otorgan importancia a todos. Luego todos los recursos expuestos son importantes. Lógicamente, esta importancia es variable y destacamos la puntuación que los encuestados le atribuyen a "internet", al "tablón de anuncios" y al "material de consulta" de orientación. Estos recursos destacan por acumular sus puntuaciones en los extremos superiores de la tabla, logrando el conjunto un promedio en importancia del 92.03\%. 
Tabla 2

Recursos generales empleados

\begin{tabular}{lccccccccccc}
\hline & \multicolumn{3}{c}{$\begin{array}{c}\text { 0= No, nada, nunca } \\
\text { 1= Sí, alguna que otra vez } \\
\text { 2= Sí, varias veces } \\
\text { 3= Sí, muchas veces }\end{array}$} & \multicolumn{4}{c}{$\begin{array}{c}\text { 0= No es importante } \\
\text { 1= Es poco importante } \\
\text { 2= Es importante }\end{array}$} \\
& 4= Sí, muchísimas veces. Siempre & \multicolumn{3}{c}{$\begin{array}{c}\text { 3= Es muy importante } \\
\text { 4= Es extremadamente importante }\end{array}$} \\
\hline PC con proyector & $\mathbf{0}$ & $\mathbf{1}$ & $\mathbf{2}$ & $\mathbf{3}$ & $\mathbf{4}$ & $\mathbf{0}$ & $\mathbf{1}$ & $\mathbf{2}$ & $\mathbf{3}$ & $\mathbf{4}$ \\
\hline Pizarra digital & 17.7 & 23.8 & 23.3 & 22.1 & 13.1 & 4.5 & 11.8 & 37.3 & 29.2 & 17.3 \\
Encerado clásico & 64.2 & 12.9 & 11.0 & 7.1 & 4.7 & 16.1 & 22.0 & 30.2 & 19.2 & 12.5 \\
Internet & 12.1 & 14.5 & 12.9 & 15.1 & 45.4 & 8.4 & 16.9 & 34.3 & 21.8 & 18.6 \\
Tablón de anuncios & 19.5 & 26.8 & 23.6 & 20.4 & 9.7 & 2.1 & 5.1 & 29.2 & 34.4 & 29.2 \\
Material de consulta & 15.4 & 23.7 & 25.0 & 20.09 & 15.0 & 2.1 & 6.7 & 27.7 & 33.9 & 29.6 \\
Cartelería & 18.3 & 29.9 & 24.2 & 16.9 & 10.6 & 2.4 & 5.5 & 25.4 & 33.6 & 33.1 \\
\hline & 27.4 & 33.7 & 24.2 & 11.1 & 3.7 & 7.0 & 13.6 & 39.6 & 26.3 & 13.5 \\
\hline
\end{tabular}

Fuente: Elaboración propia.

Recursos telemáticos empleados:

Salvo la web del instituto de secundaria de procedencia, el resto de elementos podemos considerarlos como infrautilizados, a la vista de los resultados. Así el correo electrónico, la web de orientación, el blog de orientación y el teléfono móvil obtienen un promedio de infrautilización del $64.22 \%$. Sin embargo, todos en conjunto obtienen un porcentaje de importancia del $72.56 \%$.

Individualmente destacan en importancia las webs y el correo electrónico. En menor medida el blog de orientación y el teléfono móvil (Ver Tabla 3).

Tabla 3

Recursos telemáticos empleados

\begin{tabular}{|c|c|c|c|c|c|c|c|c|c|c|}
\hline & \multicolumn{5}{|c|}{$\begin{array}{c}\text { 0= No, nada, nunca } \\
\text { 1= Sí, alguna que otra vez } \\
\text { 2= Sí, varias veces } \\
\text { 3= Sí, muchas veces } \\
\text { 4= Sí, muchísimas veces. Siempre }\end{array}$} & \multicolumn{5}{|c|}{$\begin{array}{c}0=\text { No es importante } \\
\text { 1= Es poco importante } \\
2=\text { Es importante } \\
3=\text { Es muy importante } \\
\text { 4= Es extremadamente importante }\end{array}$} \\
\hline & 0 & 1 & 2 & 3 & 4 & 0 & 1 & 2 & 3 & 4 \\
\hline $\begin{array}{l}\text { Correo electrónico } \\
\text { E-mail }\end{array}$ & 49.5 & 21.5 & 14.8 & 8.7 & 5.5 & 5.7 & 11.7 & 39.1 & 27.0 & 16.5 \\
\hline Web del Instituto & 31.4 & 26.6 & 19.6 & 12.6 & 9.8 & 6.2 & 12.9 & 37.4 & 26.3 & 17.2 \\
\hline Web de orientación & 67.9 & 16.0 & 8.5 & 4.8 & 2.9 & 8.8 & 16.0 & 35.7 & 25.1 & 14.5 \\
\hline Blog de orientación & 75.4 & 13.0 & 6.6 & 3.2 & 1.8 & 13.2 & 18.9 & 34.4 & 20.9 & 12.6 \\
\hline Teléfono móvil & 64.1 & 16.3 & 9.6 & 5.9 & 4.1 & 21.8 & 22.1 & 29.4 & 15.7 & 11.0 \\
\hline
\end{tabular}

Fuente: Elaboración propia.

Material aportado a los alumnos y alumnas: 
Existe una clara utilización de materiales tradicionales tales como fotocopias y folletos o manuales informativos, así como una clara infrautilización de otros materiales más modernos como pudieran ser los CDs con información variada. Así un 59\% de los alumnos y alumnas consultados afirman no haber recibido nunca un CD con información de orientación.

Por otro lado, el conjunto de los materiales expuestos cobran un promedio en importancia del $83.76 \%$, destacando individualmente la importancia que el alumnado concede a las tradicionales fotocopias y folletos informativos, por encima de la importancia concedida a los CDs con información variada (Ver Tabla 4).

Tabla 4

Material aportado

\begin{tabular}{|c|c|c|c|c|c|c|c|c|c|c|}
\hline & \multicolumn{5}{|c|}{$\begin{array}{c}\text { 0= No, nada, nunca } \\
\text { 1= Sí, alguna que otra vez } \\
\text { 2= Sí, varias veces } \\
\text { 3= Sí, muchas veces } \\
\text { 4= Sí, muchísimas veces. Siempre }\end{array}$} & \multicolumn{5}{|c|}{$\begin{array}{c}0=\text { No es importante } \\
1=\text { Es poco importante } \\
2=\text { Es importante } \\
3=\text { Es muy importante } \\
\text { 4= Es extremadamente importante }\end{array}$} \\
\hline & 0 & 1 & 2 & 3 & 4 & 0 & 1 & 2 & 3 & 4 \\
\hline Fotocopias & 10.2 & 19.6 & 22.7 & 25.0 & 22.5 & 2.4 & 6.9 & 33.8 & 32.1 & 24.7 \\
\hline $\begin{array}{l}\text { Folletos, manuales } \\
\text { informativos }\end{array}$ & 13.1 & 28.2 & 28.9 & 20.0 & 9.8 & 2.8 & 7.9 & 36.0 & 31.7 & 21.4 \\
\hline $\begin{array}{l}\text { CDs con } \\
\text { información variada }\end{array}$ & 59.5 & 23.0 & 8.6 & 5.9 & 3.0 & 11.2 & 17.2 & 34.0 & 23.2 & 14.4 \\
\hline
\end{tabular}

Fuente: Elaboración propia.

Horario a disposición de los alumnos y alumnas para la libre utilización del servicio de orientación:

Los datos están referidos a la posibilidad de utilización libre del servicio de orientación por parte del alumnado en los distintos horarios en los que se distribuye la jornada educativa de secundaria, y la importancia que este le otorgan a dicha franja horaria.

Tabla 5

Horarios a disposición de los alumnos y alumnas

\begin{tabular}{lccccccccccc}
\hline & $\begin{array}{c}\text { 0= No, nada, nunca } \\
\text { 1= Sí, alguna que otra vez } \\
\text { 2= Sí, varias veces } \\
\text { 3= Sí, muchas veces }\end{array}$ & \multicolumn{4}{c}{$\begin{array}{c}\text { 0= No es importante } \\
\text { 1= Es poco importante } \\
\text { 2= Es importante }\end{array}$} \\
& 4= Sí, muchísimas veces. Siempre & \multicolumn{4}{c}{$\begin{array}{c}\text { 4= Es muy importante } \\
\text { 4=xtremadamente importante }\end{array}$} \\
\hline Horario en Tutorías & $\mathbf{0}$ & $\mathbf{1}$ & $\mathbf{2}$ & $\mathbf{3}$ & $\mathbf{4}$ & $\mathbf{0}$ & $\mathbf{1}$ & $\mathbf{2}$ & $\mathbf{3}$ & $\mathbf{4}$ \\
\hline Horario en Clase Normal & 23.6 & 34.9 & 22.4 & 11.0 & 8.1 & 3.6 & 10.1 & 36.4 & 30.7 & 19.2 \\
Horario en Recreos & 30.5 & 29.1 & 21.2 & 10.2 & 9.0 & 9.2 & 15.5 & 38.5 & 23.5 & 13.2 \\
Horario Extraesc. & 29.9 & 23.1 & 19.2 & 14.0 & 13.7 & 16.6 & 15.6 & 31.9 & 21.8 & 14.1 \\
\hline & 53.6 & 19.3 & 12.8 & 8.7 & 5.6 & 21.9 & 16.0 & 30.8 & 19.2 & 12.0 \\
\hline
\end{tabular}

Fuente: Elaboración propia. 
Así, con un porcentaje promedio del $80.75 \%$, los horarios de tutorías, clase ordinaria y recreos en general, se consideran importantes para llevar a cabo labores de orientación. Sin embargo un promedio del $28 \%$ afirma no haber tenido nunca la posibilidad de utilizar el servicio de orientación en esos momentos. A esto se le suma un porcentaje medio del $29.03 \%$ que afirma haber tenido esa posibilidad alguna que otra vez.

El horario extraescolar es claramente el menos utilizado y también el menos valorado, aunque su valoración en importancia del 62\% (Ver Tabla 5).

\section{Con respecto al contraste existente entre el grado de materialización de esas actividades y/o recursos utilizados y la importancia atribuida por el alumnado a los mismos}

Del análisis comparativo entre las puntuaciones otorgadas a realidad de la orientación recibida (realidad) y su importancia (importancia), utilizando la prueba de los rangos con signo de Wilcoxon con un nivel de confianza del 95\% $(\alpha=.05)$, se obtiene en todos los casos un p-valor igual a .000 , lo que nos lleva a aceptar que existen cambios significativos en la opinión de los estudiantes. Es decir, la opinión de los estudiantes es distinta o varía significativamente según hablen de orientación recibida o de orientación ideal: las puntuaciones en importancia son significativamente más elevadas en todas las variables.

\section{Discusión y conclusiones}

Se evidencia una distancia significativa entre la importancia que la orientación académica y profesional tiene para el alumnado y el trabajo realizado por los centros educativos. Así, este último parece ser insuficiente grupal e individualmente (prácticamente inexistente el individual). Sobre este aspecto cabe mencionar a Gil (2005), en su estudio sobre la orientación e inserción laboral del alumnado de ciclos formativos de grado medio, al exponer que los departamentos de orientación cayeron en aspectos burocráticos de organización escolar, olvidándose de los problemas reales que muchas veces surgen de manera improvisada en la dinámica diaria. El autor, llega a preguntarse si la ayuda orientadora es adecuada y suficiente para conseguir los objetivos deseados por el alumnado. Desde el punto de vista del ¿Cuándo?, nuestro estudio evidencia la preferencia del alumnado para el trabajo de orientación en los horarios de tutoría y/o de clase lectiva ordinaria, cobrando especial importancia las sesiones de orientación con invitados de relevancia por su alto porcentaje de aceptación.

Montilla (2007), encuentra una vía directa de trabajo en el aula cuando se refiere a la búsqueda del compromiso del centro para decidir que materias cederán horas para intervenir los tutores y la orientadora con un programa de orientación vocacional y profesional. En la misma línea, Toscano (2004), explica que una vez aplicado su programa de orientación académica de modo directo a alumnado de bachillerato desinformado o desorientado en horario lectivo ha generado resultados positivos, considerando el alumnado receptor que la información transmitida en esas sesiones fue positiva, suficiente y significativa a efectos de, por ejemplo, el acceso a la universidad. 
Por ello cabe señalar la concordancia de estos datos con los resultados de nuestro estudio, que indican que para el alumnado es importante la introducción de un espacio horario en períodos lectivos para desarrollar contenidos propios de orientación académica y profesional. Según la importancia concedida recordamos el orden de preferencia, que es:

1. Un espacio lectivo de tutoría propio (86.3\%).

2. Un espacio lectivo en un período de otra materia $(75.3 \%)$.

3. En horario de recreo $(67.8 \%)$.

4. En horario extraescolar (62.1\%).

Por la importancia que se le concede al uso de los recursos TIC en los centros educativos, los resultados de nuestro estudio parecen concordar también con los de otros análisis realizados. Es el caso, por ejemplo, del estudio de Nogueira (2008); Sarmiento (2007); Sobrado, Ceinos, Rodicio y Ocampo (2014), o de Sobrado y Santos (2004) en su informe sobre el estado y la situación del sistema escolar en Galicia. En nuestro caso, encontramos diferencias significativas entre la importancia que tiene el uso de determinados recursos tecnológicos para el alumnado y el grado de utilización de los mismos en los centros educativos. Al respecto cabe recordar que con la excepción de internet, es insatisfactorio el grado de utilización de la mayoría de los recursos tecnológicos expuestos: correo electrónico, web y blog de orientación y teléfono móvil. Así, siendo importantes para el alumnado no se ha utilizado el correo electrónico en un $49.25 \%$ de los casos, ni la web de orientación en un $67.9 \%$ de los casos, ni el blog de orientación en un $75.4 \%$ de los casos, un $59 \%$ del alumnado no ha recibido nunca un CD con información académica y profesional.

Nogueira en el mismo estudio (2008), detectó además que los orientadores apenas utilizan las TIC en su tarea profesional, que estos poseen un nivel bajo para el manejo de recursos TIC algo avanzados (entornos de red, etc.) y un nivel medio para el uso técnico y básico de los mismos (ordenador personal y textos, presentaciones, internet y e-mail). Tienen además, poca aplicación para el diseño de recursos web de orientación. Este hecho podría explicar el poco uso de los recursos TIC manifestado por el alumnado en nuestro estudio.

Romero y Montilla (2015), recogen en su estudio con orientadores de institutos de enseñanza secundaria que una parte importante de los mismos piensa que no conoce todas las herramientas TIC que le pueden ayudar en el desempeño de la labor de orientación, así como que los cursos de formación TIC no están relacionados con su perfil profesional.

En definitiva, por lo expuesto y atendiendo a los objetivos planteados se concluye lo siguiente:

- Se necesita más actividad grupal e individual de orientación en las etapas postobligatorias, recursos técnicos variados (entrevistas individuales, test de orientación, charlas grupales con invitados de relevancia, con las familias, con el alumnado, etc.) e intervención con recursos TIC (cuya infrautilización es destacable en nuestro estudio) y convencionales (los más utilizados y muy valorados). 
- Las actividades y recursos de orientación planteados tienen gran importancia para el alumnado.

- La acción materializada por los centros satisface significativamente poco al alumnado, atendiendo a su importancia.

Dado que esta investigación se ha desarrollado en la Comunidad Autónoma de Galicia y que los servicios de orientación tienen rasgos comunes en prácticamente todo el territorio español, una posible línea de trabajo en el futuro podría ser el ampliar la investigación a todo el territorio nacional, bajo la hipótesis de que los resultados serán similares.

\section{Referencias}

Álvarez González, M. (1995). Orientación Profesional. Barcelona: Cedecs.

Álvarez González, M. (2004). Nuevos retos de la orientación en el nuevo sistema educativo. I Encuentro de Orientación y atención a la diversidad. Zaragoza: Asociación Aragonesa de Psicopedagogía.

Álvarez González, M. (2009). Modelos explicativos de la Orientación Profesional. En L. Sobrado \& A. Cortés (Coords.), Orientación Profesional. Nuevos escenarios y perspectivas (pp. 47-72). Barcelona: Biblioteca Nueva.

Álvarez Rojo, V.; García Jiménez, E.; Gil Flores, J.; Martínez Clares, P.; Romero, S. \& Rodríguez Santero, J. (2002). Diseño y evaluación de programas. Madrid: EOS.

Barreira, A. (2002). Análisis de las Competencias Profesionales de los Orientadores Escolares. (Tesis doctoral). Universidad de Santiago de Compostela. Santiago de Compostela.

Decreto 120/1998, de 23 de abril, por el que se regula la orientación educativa y profesional en la Comunidad Autónoma de Galicia. Diario Oficial de Galicia, España, 27 de abril de 1998.

García Murias, R.; Sobrado, L. \& Fernández Rey, E. (2016). Análisis de la información orientadora para la movilidad académica en el programa Erasmus. Revista Española de Orientación y Psicopedagogía, 27(1), 67-82.

Gil, G. (2005). Formación profesional, orientación e inserción laboral del alumnado de los ciclos formativos de grado medio. (Tesis Doctoral). Universitat de Valencia. Valencia.

Ley General de Educación 14/1970, de 4 de agosto. Boletín Oficial del Estado, España, 6 de agosto de 1970.

Ley Orgánica 8/1985, de 3 de julio, reguladora del derecho a la educación. Boletín Ofical del Estado, España, 4 de Julio de 1985.

Ley Orgánica 1/1990, de 3 de octubre, de Ordenación General del Sistema Educativo. Boletín Oficial del Estado, España, 4 de octubre de 1990.

Ley Orgánica 10/2002, de 23 de diciembre, de Calidad de la Educación. Boletín Oficial del Estado, España, 24 de diciembre de 2002.

Ley Orgánica 2/2006, de 3 de mayo, de Educación. Boletín Oficial del Estado, España, 4 de mayo de 2006.

Ley Orgánica, 8/2013, de 9 de diciembre, para la Mejora de la Calidad Educativa. Boletín Oficial del Estado, España, 10 de diciembre de 2013. 
Mariño, C. (2012). Análisis de los servicios de orientación educativa en España. Innovación Educativa, 22, 217-228.

Montilla, Ma .V.C. (2007). Propuesta coordinada de trabajo sobre orientación profesional y vocacional en bachillerato. Revista de Educación, 9, 133-142.

Nogueira, M. A. (2008). Diagnose da utilización e formación nas tecnoloxías da información e da comunicación dos orientadores educativos. (Tesis Doctoral). Universidade de Santiago de Compostela. Santiago de Compostela.

Ocampo, C. I. (2003). Competencias orientadoras, formación profesional e proxecto educativo municipal. Santiago: Servicio de publicación e intercambio científico, USC.

Ocampo, C. I. (2010). Proyecto docente e investigador. Universidad de Vigo (inédito).

Ocampo, C.I.; Sarmiento, J. A. \& Barreira, A. (2016). Aprendizajes y propuestas para la formación de los orientadores de secundaria a partir de la opinión del alumnado del practicum de psicopedagogía. Revista Española de Orientación y Psicopedagogía, $27(2), 8-28$.

Orden del 24 de julio de 1998 por la que se establece la organización y el funcionamiento de la orientación educativa y profesional en la Comunidad Autónoma de Galicia regulada por el Decreto 120/1998. Diario Oficial de Galicia, España, 31 de Julio de 1998.

Planas, J. A. (2009). Los servicios de orientación en las Comunidades Autónomas. En M. Álvarez González \& R. Bisquerra (Coords.), Manual de Orientación y Tutoría, versión electrónica (formato CD). Barcelona: Wolters Kluwer España.

Rodríguez Moreno, M. L. (1998). Modelos organizativos de orientación de la Unión Europea. En M. Álvarez González \& R. Bisquerra (Coords.), Manual de Orientación y Tutoría, (pp. 28-32). Barcelona: Wolters Kluwer España.

Rodríguez Moreno, M. L. (2002). Hacia una nueva orientación universitaria. Modelos integrados de acción tutorial, orientación curricular y construcción del proyecto profesional. Barcelona: UB.

Romero, C. \& Montilla, Ma. V. C. (2015). La utilización de las TIC en la orientación educativa: un estudio exploratorio sobre la situación actual de uso y formación entre los profesionales de la orientación, Revista Española de Orientación y Psicopedagogía, 26(3), 78-95.

Sánchez García, M.F. (coord.) (2013). Orientación profesional y personal. Madrid: UNED.

Sanz Oro, R. (1999). Los Departamentos de orientación en educación secundaria: roles y funciones. Barcelona: Cedecs

Sarmiento, J. A. (2007). Modelo común de orientación psicopedagógica en Galicia. Elaboración del IMMT para la representación del conceptualizaciones espistemológicas y subjetivas. (Tesis doctoral). Universidad de Vigo, Ourense.

Sobrado, L. (1999). Servicios de orientación psicopedagógica y profesional en la Unión Europea: Modelos externos e internos. En L. Sobrado (ed.), Orientación e Intervención Sociolaboral (pp. 329-350). Barcelona: Estel.

Sobrado, L. \& Barreira, A. (2012). Servicios de Orientación Educativa: organización y funcionamiento. En L. Sobrado, E. Fernández \& Maㅡ L. Rodicio (coords.), Orientación Educativa. Nuevas perspectivas (pp. 187-215). Madrid: Biblioteca Nueva.

Sobrado, L. \& Ocampo, C. I. (2000). Evaluación Psicopedagógica y Orientación Educativa. Barcelona: Estel, 
Sobrado, L. \& Santos, M.A. (Dirs.) (2004). Orientación escolar e acción titorial en Galicia. Informe sobre o estado e a situación do sistema educativo en Galicia (cursos 2000/01-2001/02). Santiago de Compostela: CEOU, Xunta de Galicia.

Sobrado, L., Ceinos, C., Rodicio, M. L. \& Ocampo, C. (2014). E-guidance for career: a new scenario of innovation and help. En J. Górna \& D. Kukla (Eds.), Vocational Counselling: changes and challenges on the labour market (pp. 210-230). Editorial Częstochowa: Akademia im. Jana Długosza w Częstochowie.

Toscano, Ma . de la O (2004). Estudio sobre la orientación académica para el tránsito del Bachillerato a la Universidad de Huelva: diseño, aplicación y evaluación de un programa de orientación para el acceso a la universidad. (Tesis Doctoral). Universidad de Huelva. Huelva.

Velaz de Medrano, C.; Manzano, N.; López Martín, E. \& Manzano Soto, N. (2013). Competencias y formación de los orientadores escolares: estudio empírico en 9 comunidades autónomas. Revista de Educación, número extraordinario, 261-292

Watts, A. G. et al. (1993). Los servicios de orientación académica y profesional en la comunidad europea (Informe-resumen del estudio realizado para la comisión de las Comunidades Europeas). Luxemburgo: Oficina de publicaciones oficiales de las Comunidades Europeas.

Fecha de recepción: 3 de marzo de 2017.

Fecha de revisión: 9 de noviembre de 2017.

Fecha de aceptación: 9 de noviembre de 2017. 
